\title{
Colonic obstruction in a tetraplegic patient: a common symptom from an uncommon cause
}

\author{
Sintip Pattanakuhar ${ }^{1} \cdot$ Apichana Kovindha ${ }^{1}$
}

Received: 12 April 2020 / Revised: 17 June 2020 / Accepted: 17 June 2020

(c) International Spinal Cord Society 2020

\begin{abstract}
Introduction It is difficult to diagnose an acute abdomen condition in people with spinal cord injury due to abnormal sensation below the injured level and multiple co-morbidities. These issues can mislead the exact diagnosis and delay proper treatment.

Case presentation A 57-year-old male with C4 AIS C tetraplegia developed nausea and vomiting, abdominal distension and feeding intolerance. Serum electrolytes indicated severe hyponatremia. A provisional diagnosis of pseudo-gut obstruction was made. After the failure of $48 \mathrm{~h}$ of conservative treatment with a nasogastric and rectal tube, abdominal CT was performed and revealed sigmoid volvulus.

Conclusions Due to the inconclusive clinical features and lack of subjective complaints, early use of CT scan or MRI is preferable in people with SCI who are suspected of an emergency intra-abdominal condition.
\end{abstract}

\section{Introduction}

It is difficult to diagnose an acute abdomen in people with spinal cord injury (SCI), who have diminished sensation below the level of injury. Multiple co-morbidities from SCI itself, as well as from the treatment can mislead the exact diagnosis and delay proper treatment. Here, we present the case of a man with tetraplegia who suffered from symptoms of abdominal obstruction. The diagnosis of sigmoid volvulus (SV) was delayed due to the misleading of abnormal serum electrolytes. We also review the condition of SV and discuss the practical points for the diagnosis of an acute abdomen condition in people with SCI.

\section{Case presentation}

A 57-year-old male with a 4-year-history of C4 AIS C tetraplegia was admitted to the rehabilitation ward for managing multiple grade 4 pressure ulcers on his coccyx

Sintip Pattanakuhar

umedicus@hotmail.com

1 Department of Rehabilitation Medicine, Faculty of Medicine, Chiang Mai University, Chiang Mai, Thailand and back. During the admission, he was also diagnosed with major depressive disorder and was treated by sertraline $25 \mathrm{mg} /$ day and lorazepam $1 \mathrm{mg}$ before bedtime. His bowel management was using hypertonic sodium chloride enema 20-40 $\mathrm{ml}$ per times, three times a week. He reported no bowel incontinence or fecal impaction for the year before admission. Bladder management was indwelling catheter with daily urinary output more than two liters. Other medications were baclofen $30 \mathrm{mg} /$ day and oxybutynin $15 \mathrm{mg} /$ day. One week after admission, the man developed nausea and vomiting, abdominal distension and feeding intolerance. Mild, poorly localized abdominal pain (numeric rating scale $=2$ ) was reported. No autonomic dysreflexia was present. Physical examination revealed normoactive bowel sounds and digital rectal examination revealed an empty rectum. Serum electrolytes indicated severe hyponatremia $(\mathrm{Na}=98 \mathrm{mmol} / \mathrm{dl})$ and mild hypokalemia $(\mathrm{K}=3.3 \mathrm{mmol} /$ dl). A plain abdominal $\mathrm{x}$-ray demonstrated severe dilation of the entire colon with the absence of rectal gas shadow (Fig. 1). A distal colonic obstruction was provisionally diagnosed. Surgical consultation was performed and a provisional diagnosis of pseudo-gut obstruction due to electrolyte imbalance was provided by the consultant. Conservative treatments with the nasogastric and rectal tube were recommended, as well as intravenous ceftriaxone and metronidazole. After $48 \mathrm{~h}$ of conservative treatment, signs of peritonitis were detected. Emergency abdominal CT was 


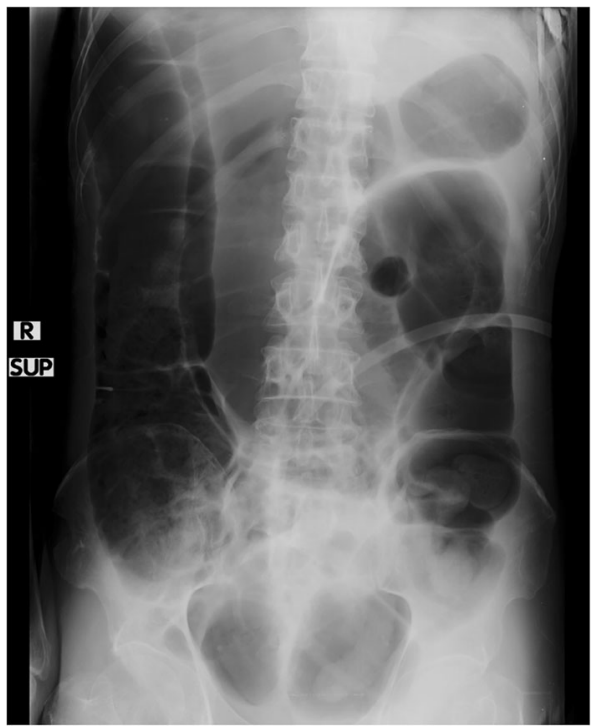

Fig. 1 Plain abdominal X-ray, supine position, revealed severe dilation of the entire colon with the absence of rectal gas shadow.

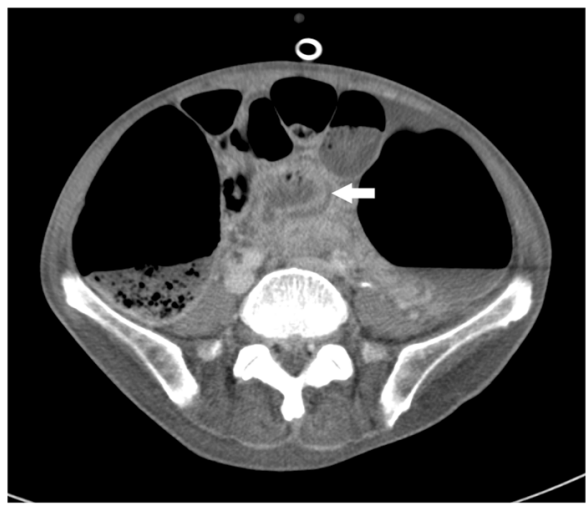

Fig. 2 Abdominal CT, cross-sectional plane, demonstrated a whirled soft tissue mass at a lower abdomen (arrow), compatible with sigmoid volvulus.

requested. It revealed a whirled soft tissue mass at the lower abdomen, compatible with sigmoid volvulus (SV) with patent ileocaecal valve (ICV) (Figs. 2, 3). Flexible sigmoidoscopy was performed, showing SV at $25 \mathrm{~cm}$ from the anal valve. Endoscopic sigmoid detorsion failed, therefore, open sigmoid detorsion with loop transverse colostomy was performed. The man was transferred back to rehabilitation on day 3 after surgery and serum sodium was normal (140 mEq) 7 days after the withdrawal of sertraline.

\section{Discussion}

Colonic obstruction is not uncommon in people with SCI [1]. One of the potential causes is SV. From a literature

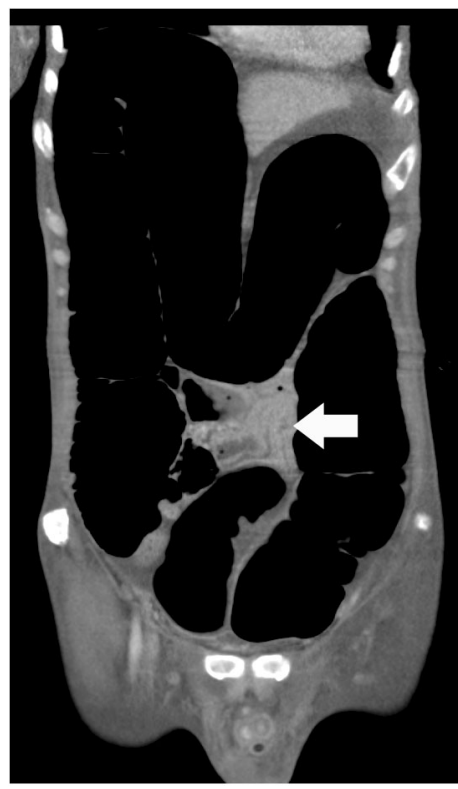

Fig. 3 Abdominal CT, coronal plane, demonstrated a whirled soft tissue mass at a lower abdomen (arrow), compatible with sigmoid volvulus.

review, the incidence of colonic (caecal and sigmoid) volvulus in people with SCI is $2.6 \%$ [2]. SCI per se is not the major predisposing factor of $\mathrm{SV}$ but it increases the incidence of risk factors such as immobility, chronic constipation causing fecal overloading and chronic use of anthraquinone laxative (e.g. senna) causing degeneration of the myenteric plexus and acontractile colon [2]. Duration of injury, use of the anti-spastic or anti-depressive drugs have also suspected to be risk factors [2]. Symptoms of SV in people with SCI are abdominal discomfort, feeding intolerance, poorly localized abdominal pain and frequent autonomic dysreflexia [2]. Physical examination usually shows signs of abdominal distension and generalized tender with or without peritonitis. Differences between the characteristics of bowel sounds, which are present above but absent below an obstruction level, may additionally be an important clinical clue. However, this may not be a reliable clinical sign since it may be difficult to detect. The most important signs that indicate SV are empty rectum during rectal examination and absence of fecal mass in the left lower quadrant of the abdomen $[2,3]$. Plain abdominal X-ray finding depends on the competency of ICV. Severe dilation limited in the colon will be observed when ICV is competent and generalized dilation of small bowel and colon will be detected when $\mathrm{ICV}$ is incompetent. Moreover, plain abdominal X-ray findings may also demonstrate an absence of rectal gas shadow [3]. The classical coffee bean sign is seen in $<60 \%$ of cases [3] and a bird-beak appearance or corkscrew mucosal pattern of barium enema increases the diagnostic 
yield by $20-30 \%$ [3]. At present, CT or MRI abdomen showing a whirled soft tissue mass has $100 \%$ accuracy [3].

Management of SV is dependent on the presence or absence of complications such as colonic gangrene or perforation and condition of the patient. If colonic gangrene or perforation presents, emergency laparotomy with simple distortion with a colostomy, emergency resection with a colostomy or emergency resection and primary anastomosis (ERPA) must be performed, depending on the condition of the patient and the viability of the tissue [3]. If colonic gangrene or perforation is absent, reduction by flexible sigmoidoscopy followed by elective surgery to prevent recurrence after endoscopy is preferred [3]. If reduction by flexible sigmoidoscopy fails, an emergency laparotomy is needed. The mortality rate of SV is below $10 \%$ in the overall population [3], but one case series of individuals with SCI population showed a mortality rate of $12 \%$ [2].

Other differential diagnoses for acute abdomen in people with SCI are acute appendicitis, acute gastrointestinal (GI) bleeding, perforated peptic ulcer, gallstones, renal stones and ectopic pregnancy. Regarding acute appendicitis, the most common clinical manifestation is abdominal distension and/or abdominal discomfort (62\%), followed by fever (15\%) [4]. Abdominal CT is a useful investigation for confirming the diagnosis [4]. However, due to unspecific clinical manifestations, $92 \%$ of people with SCI who diagnosed with acute appendicitis developed ruptured appendicitis, resulting in the 30-day mortality rate of $4 \%$ [4]. Acute GI bleeding in people with SCI were mostly found in an acute phase and might be associated with methylprednisolone [5]. Clinical manifestations are not specific, including anorexia, hematemesis and passing melena [6]. When indicated, early GI endoscopy is a beneficial tool for both diagnostic and therapeutic purposes [6]. Perforated peptic ulcer in people with SCI was reported especially in acute phase and associated with using methylprednisolone [5]. The diagnosis may be delayed, resulting in fatal complications such as sepsis and death [5]. The prevalence of gallstones in people with SCI 17-31\% [1]. Risk factors are diabetes, obesity and family history of gallbladder disease [1]. Symptoms and signs may be atypical or even absent then the pathologic processes are often advanced at diagnosis [1]. Fever might present if acute cholecystitis is developed. Abdominal ultrasonography is a useful investigation tool and open or laparoscopic prophylactic cholecystectomy is a treatment of choice with the comparable mortality rate to the normal population [1]. Renal stones are not uncommon as a prevalence of $14.8 \%$ in people with SCI [7]. It is associated with neurogenic bladder dysfunction and vesicoureteric reflux [7]. Although the symptoms are unspecific, urine examination showing hematuria could be an important diagnostic clue [7]. An acute abdomen in people with SCI resulting from ruptured ectopic pregnancy has been reported. The diagnosis was difficult due to lack of classical signs and symptoms [8]. Therefore, in women with SCI who develop an acute abdomen during reproductive age, urine pregnancy test might be diagnostically beneficial for excluding obstetrical conditions.

In this case report, the diagnosis of SV was delayed due to a focus on abnormal serum electrolytes. Sertralineinduced severe hyponatremia is common [9], and it was suspected in this case. Hyponatremia, as well as hypokalemia, can cause pseudo-gut obstruction [10], but in both conditions, bowel sounds should be decreased [11]. In this case, it is difficult to determine whether sertraline was a potential cause of the hyponatremia that resulted in colonic dysmotility and predisposed the patient to have SV or the patient had hyponatremia due to electrolyte abnormalities associated with SV by itself without any effect from sertraline. Fecal impaction is part of the differential diagnosis of gut obstruction in people with SCI, but digital rectal examination should reveal fecal content in the rectum. Finally, the most important point in this case is early use of CT scan or MRI is preferable in people with SCI who present with possible signs (vomiting, abdominal distention, feeding intolerance) of an acute abdomen.

\section{Compliance with ethical standards}

Conflict of interest The authors declare that they have no conflict of interest.

Ethical standards This case report received an exemption from review by the Institutional Ethics Committee of the Faculty of Medicine, Chiang Mai University, Chiang Mai, Thailand.

Publisher's note Springer Nature remains neutral with regard to jurisdictional claims in published maps and institutional affiliations.

\section{References}

1. Ebert E. Gastrointestinal involvement in spinal cord injury: a clinical perspective. J Gastrointestin Liver Dis. 2012;21:75-82.

2. Fenton-Lee D, Yeo BW, Jones RF, Engel S. Colonic volvulus in the spinal cord injured patient. Paraplegia. 1993;31:393-7.

3. Raveenthiran V, Madiba TE, Atamanalp SS, De U. Volvulus of the sigmoid colon. Colorectal Dis. 2010;12:e1-17.

4. Strauther GR, Longo WE, Virgo KS, Johnson FE. Appendicitis in patients with previous spinal cord injury. Am J Surg. 1999; 178:403-5.

5. Lee HC, Cho DY, Lee WY, Chuang HC. Pitfalls in treatment of acute cervical spinal cord injury using high-dose methylprednisolone: a retrospect audit of 111 patients. Surg Neurol. 2007;68: S37-41.

6. Joseph RE, Epsten R, Kowlessar OD. An unusual cause of GI bleeding in a quadriplegic: report of a case and review of the literature. J Natl Med Assoc. 1982;74:83-5. 
7. Hall MK, Hackler RH, Zampieri TA, Zampieri JB. Renal calculi in spinal cord-injured patient: association with reflux, bladder stones, and foley catheter drainage. Urology. 1989;34:126-8.

8. Rawal N, Sharma S, Soni B. A case of silent rupture of an ectopic pregnancy. Spinal Cord. 2008;46:758-9.

9. Viramontes TS, Truong H, Linnebur SA. Antidepressant-induced hyponatremia in older adults. Consult Pharm. 2016;31:139-50.
10. Jetmore AB, Timmcke AE, Gathright JB Jr., Hicks TC, Ray JE, Baker JW. Ogilvie's syndrome: colonoscopic decompression and analysis of predisposing factors. Dis Colon Rectum. 1992;35:1135-42.

11. Sunnoqrot N, Reilly RF. Hypokalemia associated with colonic pseudo-obstruction (ogilvie's syndrome). Case Rep Nephrol Dial. 2015;5:118-23. 